\title{
Efficient Flooding Scheme Based on 1-hop Information in Mobile Ad Hoc Networks
}

\author{
Hai Liu, Pengjun Wan, Xiaohua Jia, Xinxin Liu, and Frances Yao \\ Dept of Computer Science \\ City University of Hong Kong, HongKong \\ \{liuhai@cs, pwan@cs,jia@cs, whulxx@, csfyao@\}cityu.edu.hk
}

\begin{abstract}
Flooding is one of the most fundamental operations in mobile ad hoc networks. Traditional implementation of flooding suffers from the problems of excessive redundancy of messages, resource contention, and signal collision. This causes high protocol overhead and interference to the existing traffic in the networks. Some efficient flooding algorithms were proposed to avoid these problems. However, these algorithms either perform poorly in reducing redundant transmissions, or require each node to maintain 2 -hop (or more) neighbors information. In the paper, we study the sufficient and necessary condition of $100 \%$ deliverability for flooding schemes that are based on only 1-hop neighbors information. We further propose an efficient flooding algorithm that achieves the local optimality in two senses: 1) the number of forwarding nodes in each step is the minimal; 2) the time complexity for computing forwarding nodes is the lowest, which is $O(n \log n)$, where $n$ is the number of neighbors of a node. Extensive simulations have been conducted and simulation results have shown that performance of our algorithm is significantly better than the existing message efficient flooding methods.
\end{abstract}

Keywords-flooding, broadcasting, mobile ad hoc networks, wireless networks

\section{INTRODUCTIONS}

Flooding is one of the most fundamental operations in mobile ad hoc networks (MANET). Most of the major routing protocols, such as DSR [1], AODV [2], ZRP [3], LAR [4], etc., rely on flooding for disseminating route discovery, route maintenance, or topology update packets. Flooding is a very frequently invoked utility function in MANETs. Therefore, an efficient implementation of flooding scheme is crucial in reducing the overhead of routing protocols and improving the throughput of networks.

Pure flooding, or called blind flooding, was first discussed in $[5,6]$, where every node in the network retransmits the flooding message when it is its first time to receive it. This simple scheme guarantees that a flooding message can reach all nodes if there is no collision and the network is connected. However, it generates excessive amount of redundant network traffic, because all nodes in the network transmit the flooding message. This will consume a lot of energy resource of mobile nodes and cause the congestion of the network. Furthermore, due to the broadcast nature of radio transmissions, there is a

This work is supported in part by Research Grants Council of Hong Kong under grant numbers CityU 1165/04E and CityU 114505. very high probability of signal collisions when all nodes flood the message in the network at the same time, which would cause more re-transmissions or some nodes failing to receive the message. It is so called the broadcast storm problem [7]. Sinha et al claimed that "in moderately sparse graphs the expected number of nodes in the network that will receive a broadcast message was shown to be as low as $80 \%$ " in [8].

To solve the broadcast storm problem, several schemes have been proposed to reduce the redundancy in flooding operations. The most notable works are [9], [10], and [11]. However, these algorithms either perform poorly in reducing redundant transmissions, or require each node to maintain 2hop neighbor information. Maintaining 2-hop neighbor information for each node incurs extra overhead of the system and the information can be hardly accurate when the mobility of the system is high. In the paper, we propose an efficient flooding algorithm that is only based on 1-hop neighbors information, which makes the protocol easy to be implement and light-weight in overhead. Our proposed algorithm also achieves the local optimality in two senses: 1) the number of forwarding nodes is the minimal; 2) the time complexity is the lowest. Time complexity for computing the forwarding nodes in each step is $O(n \log n)$, which is the lower bound ( $n$ is the number of neighbors of a node).

Efficient flooding scheme is different from the broadcast mechanisms discussed in $[12,13]$. The broadcast mechanism is used for transmission of large amount data or stream media data, which requires a broadcast routing to find an efficient route before the actual transmission of data, so that data can be transmitted efficiently along the pre-found route. In contrast, flooding is usually used for dissemination of control packets, which is a one-off operation. It does not need routing before hand.

\section{RELATED WORK}

The existing efficient flooding schemes can be classified into three categories based on the information each node keeps: 1) no need of neighbor information; 2) 1-hop neighbor information; 3) 2-hop or more neighbor information.

Schemes in the first category do not need information of neighbors. Pure flooding scheme is a typical example in this category. Authors in [7, 14] showed the serious problem that pure flooding causes through analysis and simulations. A probabilistic-based scheme was further proposed to reduce 
redundant rebroadcasts and differentiate timing of rebroadcasts to avoid collisions. Upon receiving a flooding message for the first time, a node will forward it with probability $P$. Clearly, when $P=1$, this scheme is equivalent to pure flooding. The probabilistic scheme includes counter-based, distance-based, location-based and cluster-based flooding schemes. Simulation results showed different levels of improvement over pure flooding. This probabilistic scheme was further investigated in [15]. It showed that the success rate curve for probabilistic flooding tends to become linear for the network with low average node degree, and resembles a bell curve for the network with high average node degree. In these schemes, a non-redundant transmission might be dropped out, without being forwarded further. This will cause some nodes in the network failing to receive the flooding message (i.e., these nodes are not reached by the flooding). Besides this deliverability problem, another major concern of these techniques is the difficulties in setting the right threshold value (e.g., retransmission probability, etc.) in various network situations [16].

Schemes in the second category assume that each node keeps information of 1-hop neighbors. 1-hop neighbor information can be obtained by exchanging the HELLO message in MAC layer protocols. A major issue in the schemes that use 1-hop or 2-hop information is the selection of a subset of neighbors for forwarding the flooding message. There are two strategies for choosing forwarding nodes: sender-based, where each sender nominates a subset of its neighbors to be the next hop forwarding nodes, and receiver-based, where each receiver of a flooding message makes its own decision on whether it should forward the message. Several flooding schemes that use 1-hop information and guarantee $100 \%$ deliverability were discussed in [10]. This work also analyzed the performance of the two strategies for choosing forwarding nodes. To avoid transmission collision, it also proposed a simple transmission order for forwarding nodes: a farther neighbor waits for a shorter time to forward a message after it receives it. The flooding with self pruning (FSP) scheme proposed in [17] is a receiver-based scheme that uses 1-hop information. In this scheme, a sender forwards a flooding message by attaching all of its 1-hop neighbors to the message. A receiver compares its own 1-hop neighbors with the node list in the message. If all its 1-hop neighbors are already included in the list, it will not forward the message; otherwise it forwards the message as its sender. The work in [18] compared the performance of several flooding schemes. It showed that the improvement of FSP is very limited in most of network conditions. Another notable work of efficient flooding that uses 1-hop neighbor information is Edge Forwarding [9]. For each node, its transmission coverage is partitioned into six equalsize sectors. A node, upon receiving a flooding message, makes its own decision whether it should forward the message based on the availability of other forwarding nodes in the overlapped areas. Taking an example in Fig. 1, node $a$, whose coverage disk is partitioned into six sectors, floods a message that is received by its neighbor $b$. Node $b$ dose not need to forward the message if and only if 1) there exist nodes in the small enclosed areas $A, B$ and $C$; and 2) Any nodes in areas $D$ and $E$ can be reached by the nodes in $A$ and $C$, respectively. This is because the coverage disk of $b$ can be covered by either $a$ or the nodes in areas $A, B$, and $C$. By doing so, it reduces the forwarding nodes in flooding.

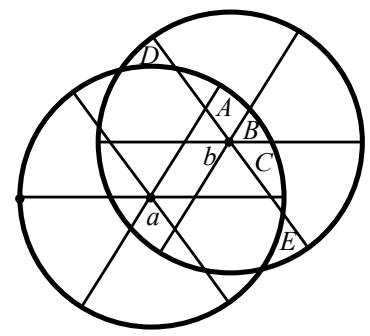

Fig. 1. Example of Edge Forwarding.

Most existing flooding schemes that use neighborhood knowledge are based on information of 2-hop neighbors. To obtain the information about 2-hop neighbors, one solution is that each node attaches the list of its own neighbor information to the HELLO message for exchange. The schemes proposed in $[17,19,20,21]$ are sender-based, while schemes in $[11,22,23,24,25,26]$ are receiver-based. In the schemes that use 2-hop neighbor information, each node knows the network topology (connectivity) of 2-hop neighbors. To forward messages efficiently, the task for each node is to select the minimal subset of its 1-hop neighbors that can reach all its 2-hop neighbors. A multipoint relaying method was proposed in $[19,20]$, which tries to find the minimal number of forwarding nodes among the neighbors. Finding the minimal number of forwarding nodes was proved to be NPcomplete [20]. Authors proposed a heuristic algorithm that selects forwarding nodes at each step, such that the number of newly covered neighbors is maximized. The approximation ratio of this heuristic algorithm was proved to be at most logn, where $n$ is the number of 2-hop neighbors. Notice that this performance ratio is only for each step (i.e., for 2-hop neighbors), not for the entire network. Another important technique is the use of connected dominating set (CDS) [11, 27]. A dominating set (DS) is a subset of nodes such that every node in the graph is either in the set or is adjacent to a node in the set. A CDS is a connected DS. Any routing in MANETs can be done efficiently via CDS [11]. Although finding minimal CDS (MCDS) is NP-hard even in unit disk graph (UDG) [28], some distributed algorithms for computing MCDS with approximation ratio have been proposed in [27, 29]. However, maintaining a CDS in the network is costly, which is not suitable for flooding operations in highly mobile situations. Generally, the schemes that use 2-hop neighbor information incur high protocol overhead in the network with high mobility and high node density, and they cannot be easily fitted into a network that does not support 2-hop neighbor information exchange.

Our flooding scheme requires each node to keep only 1hop neighbor information. We prove that our flooding scheme not only guarantees $100 \%$ deliverability, but also achieves the local optimality in terms of number of forwarding nodes and 
computational complexity. In this paper, we will not discuss the scheduling of transmissions of forwarding nodes. Interested readers can refer to the related work in [30, 31].

The rest of the paper is organized as follows. We propose an efficient flooding scheme in section 3. Section 4 discusses the handling of mobility. In section 5, we discuss the simulation of our flooding scheme by using $n s-2$ test-bed and compare its performance with other flooding algorithms. Finally, we conclude the work in section 6.

\section{EFFicient FloOding SCHEME BASEd ON 1-HOP INFORMATION}

\subsection{System Model and Overview of Method}

We assume all nodes in the network have the same transmission range $R$. Thus, the network can be represented as a unit disk graph $G(V, E)$. We assume the network is connected. Each node $v$ in $V$ has a unique ID, denoted by $i d(v)$. Let $N(v)$ denote the set of neighbor nodes of $v$. That is, nodes in $N(v)$ are within the transmission range of $v$ and can receive signals transmitted by $v$. Node $v$ needs to know the information of its neighbors, including their IDs and their geographic locations. The 1-hop neighbor information can be easily obtained from the HELLO messages periodically broadcasted by each node. For the rest of the paper, we simply use neighbors to mean 1hop neighbors.

The basic idea of our flooding scheme is as follows. When a node (called the source) has a message to be flooded out, it computes a subset of its neighbors as forwarding nodes and attaches the list of the forwarding nodes to the message. Then, it transmits (broadcasts) the message out. After that, every node in the network does the same as follows. Upon receiving a flooding message, if the message has been received before, it is discarded; otherwise the message is delivered to the application layer, and the receiver checks if itself is in the forwarding list. If yes, it computes the next hop forwarding nodes among its neighbors and transmits the message out in the same way as the source. The message will eventually reach all the nodes.

We discuss our method in three parts: a) forwarding node selection, where a node selects a subset of its 1-hop neighbors to forward the flooding message; b) forwarding node optimization, which further reduces the size of forwarding nodes by removing the nodes that are already covered; c) mobility handling, where each node incrementally updates its forwarding set in response to topology changes.

\subsection{Theoretical Foundations of Minimal Forwarding Nodes}

We aim at designing a 1-hop flooding scheme. Flooding schemes in [9], [10] and [17] are all 1-hop flooding schemes that guarantee $100 \%$ deliverability of flooding messages. To achieve the optimal efficiency, we need to study the sufficient and necessary condition of $100 \%$ deliverability for flooding schemes that are based on 1-hop information. We introduce the following definitions.

Def 1. Coverage disk of a node. The coverage disk of node $s$, denoted by $d(s)$, is a disk that is centered at $s$ and whose radius is the transmission range of $s$.

Since all neighbors of node $s$ should be covered by $d(s)$, in this paper, we call " $s$ covers $u$ " or " $u$ is covered by $s$ " when $u$ is a neighbor of $s$.

Def 2. Coverage area of a node-set. The coverage area of a set of nodes $A$, denoted by $C(A)$, is the union of coverage disks of nodes in $A$.

We simply call "the area is covered by $A$ " if the area is within $C(A)$.

Def 3. Neighbor's coverage area. The neighbor's coverage area of node $s$ is the union of coverage disks of all $s$ 's neighbors plus $s$ itself, i.e., $C(N(s) \cup\{s\})$.

Def 4. Boundary of neighbor's area. The boundary of neighbor's area of node $s$ is the boundary of the area of $C(N(s) \cup\{s\})$.

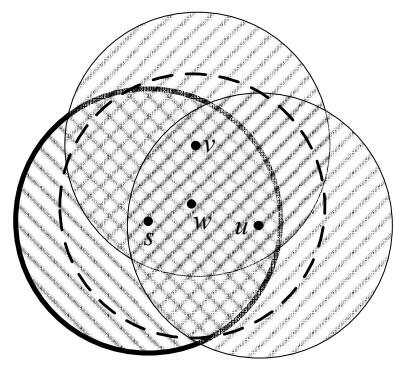

Fig. 2. Neighbor's area of node $s$.

For simplicity, the neighbor's coverage area is called neighbor's area and the boundary of neighbor's area called neighbor's boundary for the rest of the paper. For example in Fig. 2, the set of neighbors of $s N(s)=\{u, v, w\}$. Thus, the neighbor's area of $s$ is $C(\{s, u, v, w\})$, i.e., whole shadow area. The neighbor's boundary of $s$ is the outside boundary of this shadow area.

Def 5. Forwarding set. The set of forwarding nodes of $s$, denoted by $F(s)$, is a subset of $s$ 's neighbors that are selected for forwarding the flooding message $(F(s)$ includes $s$ itself).

Def 6. Minimum forwarding set $F_{\min }(s)$. The minimal forwarding set of $s$, denoted by $F_{\text {min }}(s)$, is the smallest $F(s)$ that covers the neighbor's area of $s$.

Theorem 1. A 1-hop flooding scheme achieves $100 \%$ deliverability if and only if for each node $s$ the neighbor's area of $s$ is covered by $F(s)$.

Proof. Sufficient condition $(\leftarrow)$. Suppose for each node $s$ the neighbor's area of $s$ is covered by $F(s)$. We need to prove that the flooding scheme achieves $100 \%$ deliverability.

For each transmission node $s$, since all 2-hop neighbors of $s$ are within the neighbor's area of $s$, they are sure to be 
covered by nodes in $F(s)$. Thus, all nodes that are 2-hop away from the source $s$ are sure to be covered by $F(s)$. Notice that $s$ 's 3-hop neighbors are neighbors of $s$ 's 2-hop neighbors. There must exist some transmission nodes in $F(s)$, such that $s$ 's 3-hop neighbors are 2-hop neighbors of these transmission nodes. Thus, s's 3-hop neighbors are sure to be covered by forwarding sets of these transmission nodes. Nodes that are 4hop and more from the source can be proved in the similar way. Therefore, the flooding message will be forwarded hop by hop throughout the whole network.

Necessary condition $(\rightarrow)$. Suppose a flooding scheme $A$ achieves $100 \%$ deliverability. Let $F_{A}(s)$ denote the set of forwarding nodes of $s$ that is computed by $A$. We need to prove that for each node $s$ the neighbor's area of $s$ is covered by $F_{A}(s)$. We prove it by contradiction.

We consider a kind of networks where all nodes are within coverage disk of a central node, denoted by $s$. That is, any network in this category consists of a central node and its neighbors, shown in Fig. 3. Suppose scheme $A$ does not guarantee that for each node $s$ the neighbor's area of $s$ is covered by $F_{A}(s)$. There must exist such a network as shown in Fig. 3 and the neighbor's area of $s$ is not fully covered by $F_{A}(s)$. Since $F_{\min }(s)$ is the smallest forwarding set that covers the neighbor's area of $s$, we have $F_{\min }(s) \not \subset F_{A}(s)$. In other words, there exists node $u \in F_{\min }(s)$ and $u \notin F_{A}(s)$. Notice that coverage disks of all nodes in $F_{\min }(s)$ are sure to contribute to the neighbor's boundary of $s$ (if not, it can be removed from $F_{\text {min }}(s)$ ). We place node $v$ on the boundary that is contributed only by $u$ (dashed line in Fig. 3 is the neighbor's boundary of $s$ ). Notice that $s$ has information of only 1-hop neighbors. $s$ does not know that $v$ is a neighbor of $u$ and $u$ is the only node to reach $v$. So $v$ can not be covered by any node in $F_{A}(s)$ since $u \notin F_{A}(s)$.

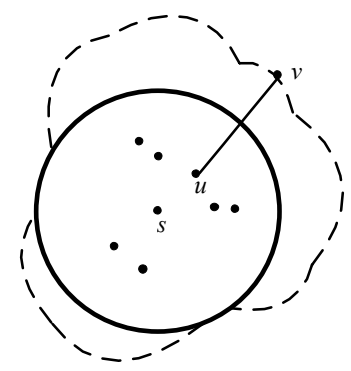

Fig. 3. Example of neighbor's area of $s$.

On the other hand, since there is no other nodes outside coverage disk of $s$, node $v$ can neither be covered by forwarding set of other nodes. That is, node $v$ will eventually miss the flooding message. It contradicts the assumption that flooding scheme $A$ achieves $100 \%$ deliverability. Theorem 1 is proved.

Theorem 1 tells us that the sufficient and necessary condition of $100 \%$ deliverability for any 1-hop flooding scheme is that for each node $s$, the neighbor's area of $s$ should be covered by $F(s)$. Otherwise, some nodes in the network may miss the flooding message. Theorem 1 gives the theoretical guideline for computing $F(s)$ in our flooding scheme.

\subsection{Computing Minimal Forwarding Nodes}

Suppose $s$ is a node that receives a flooding message for the first time and $s$ appears in the forwarding list attached to the message ( $s$ could be the original source of the message). $s$ is designated as a forwarding node and it computes the next hop forwarding nodes from its neighbors. Since $s$ only has 1hop neighbor information, it does not know who are the 2-hop neighbors. To achieve $100 \%$ deliverability, according to Theorem 1, $F(s)$ must cover the entire neighbor's area of $s$. Our task can be formally defined as:

$$
\text { Minimize } F(s) \text { such that } \bigcup_{v \in F(s)} d(v)=\bigcup_{u \in N(s)} d(u) \text {. }
$$

Taking the example in Fig. 2 again, $s$ has three neighbors: $u, v$ and $w$. Since $d(u) \cup d(v) \cup d(s)$ makes up the neighbor's area of $s$, it is enough to cover all s's 2-hop neighbors if only $u$ and $v$ forward the message. In the other word, $d(w) \subseteq d(u) \cup d(v) \cup d(s)$, there is no need for $w$ to forward the message.

To minimize $F(s)$, every node in $F(s)$ must contribute to the neighbor's boundary of $s$; otherwise, this node can be removed from $F(s)$ without affecting the coverage area of $F(s)$. Therefore, computing the minimal $F(s)$ is to find a subset of $N(s)$ such that every node in the subset contributes to the neighbor's boundary of $s$.

We first give a simple $O\left(n^{2}\right)$ algorithm to compute $F(s)$ as follows, where $n=|N(s)|$. Since the outside nodes of $N(s)$, i.e., the nodes further away from $s$, are usually the nodes that contribute to the neighbor's boundary of $s$, all nodes in $N(s)$ are sorted in descending order into a list according to their Euclidean distance to $s$. The first node in the list (that is the farthest away from $s$ ) is included in $F(s)$. Each time, the next node in the list is considered. If its coverage disk is not fully covered by the so far constructed $F(s)$, it is added into $F(s)$. This operation is repeated until all nodes in the list are considered. It is not difficult to see that $F(s)$ is the minimum (i.e., every node in $F(s)$ contributes to the neighbor's boundary of $s$ ) and the time complexity is $O\left(n^{2}\right)$.

The next we present an algorithm with time complexity $O(n \log n)$. The strategy of this method is to compute the neighbor's boundary of $s$, and thus the nodes that contribute to this boundary are the nodes in $F(s)$. We use the pair-wise boundary merging method to compute the boundary efficiently. Initially, each node is arbitrarily paired with another node to merge their coverage boundaries. Then, the merged pair's boundary is further merged with another pair's boundary. This merge operation is repeated until eventually there is only one big merged boundary, which is the neighbor's boundary of $s$. The minimal $F(s)$ consists of the nodes that contribute to this boundary. 


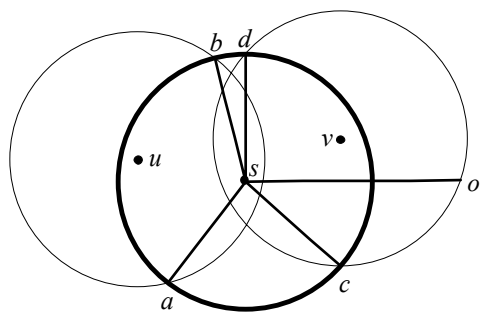

Fig. 4. Example of arcs.

Before considering the procedure for merging boundaries, we introduce data structures to represent arcs and boundaries. A boundary consists of a sequence of arcs. If we use the location of $s$ as the reference point, any arc in the neighbor's boundary of $s$ can be uniquely defined by a 3-tuple $\left(\theta^{s}, u, \theta^{e}\right)$, where $\theta^{s}, u$ and $\theta^{e}$ are the starting angle, the centre and the ending angle of the arc, respectively. $\theta^{s}$ and $\theta^{e}$ are relative to the horizontal line going through $s$ counting in anti-clock direction. For example in Fig. 4, line os is the horizontal line from $s$, which is used as the reference line in counting starting and ending angles of arcs. Arc $a b$ of disk $u$ is represented by ( $\angle o s b, u, \angle o s a$ ), where $\angle o s b$ is the starting angle and $\angle o s a$ the ending angle of $a b$.

A boundary consists of a sequence of arcs. Thus, a boundary is represented by an array of arcs, denoted by $B[]$. The $i$ th element in $B[], B[i]=\left(\theta_{i}^{s}, u_{i}, \theta_{i}^{e}\right), i=1, \ldots, m$, denotes the $i$ th arc in the boundary. The arcs in $B[]$ are sorted in nondescending order according to their starting angles. That is, $B[1] . \theta_{1}^{s} \leq B[2] . \theta_{2}^{s} \leq \ldots \leq B[m] . \theta_{m}^{s}$. This sorted feature of arcs in $B$ is critical to the efficient merging algorithm to be presented below. Because of this feature, the arcs in two boundaries can be merged in the same sequential order as the progress of their array indices without backtracking. To make the ending angle greater than the starting angle, any arc that crosses the horizontal line from $s$ is split into two arcs. For the same example in Fig. 4, arc $c d$ is split into arcs $b d$ and $c o$ and they are represented by $\left(0^{\circ}, v, \angle o s d\right),\left(\angle o s c, v, 360^{\circ}\right)$, respectively. The neighbor's boundary of $s$ in Fig. 4 can be represented as $B[]=\left\{b d, d b, b a, a c, c_{o}\right\}=\left\{\left(0^{\circ}, v, \angle o s d\right)\right.$, $(\angle o s d, s, \angle o s b),(\angle o s b, u, \angle o s a),(\angle o s a, s, \angle o s c),(\angle o s c, v$, $\left.\left.360^{\circ}\right)\right\}$.

Considering merging two boundaries $B_{i}$ and $B_{j}$ into a new one $B$, we start from the first arcs in $B_{i}$ and $B_{i}$, respectively, merge them and store the merged arc in $B$. Suppose now we are at the point of merging the $k^{\text {th }}$ arc of $B_{i}$ (i.e., $\left.B_{i}[k]\right)$ with the $l^{\text {th }}$ arc of $B_{j}$ (i.e., $B_{j}[l]$ ), and storing the merged arc in $B[h]$. Notice that two arcs intersect with each other at no more than two points (because any two different disks intersect with each other at no more than two points). There are three possible cases of the intersection: 1) no intersection; 2) only one intersecting point; 3 ) two intersecting points. We discuss the cases one by one.
If $\operatorname{arcs} B_{i}[k]$ and $B_{j}[l]$ have no intersection, it can be further divided in three sub-cases: 1.1) the sectors of two arcs are overlapped with each other, as in Fig. 5(a);1.2) sector of one arc is contained by the other, as in Fig. 5(b); 1.3) There is no overlapping of the sectors of two arcs, in Fig. 5(c). For case 1.1 , arc $B_{i}[k]$ contributes to the resulting boundary $B$. Thus, the resulting arc in $B$ is $B_{i}[k]$, i.e., $B[h]=B_{i}[k]$. Then, we move to next arc in $B_{i}$ and compare $B_{i}[k+1]$ with $B_{j}[l]$. For case 1.2, segment $a b$ of $B_{i}[k]$ is for sure to contribute to $B$, but segment $b c$ may intersect with arc of $B_{j}[l+1]$. Therefore, $B[h]$ is set to $a b$. Then, we move to next $\operatorname{arc}$ in $B_{j}$, i.e., $B_{j}[l+1]$, to compare it with segment $b c$. For case 1.3 , without losing generality, assuming $B_{i}[k] . \theta^{s}<B_{j}[l] . \theta^{s}$, arc $B_{i}[k]$ is for sure to contribute to $B$, but $B_{j}[l]$ may intersect with $B_{i}[k+1]$. We set $B[h]=B_{i}[k]$, and move the next to compare $B_{i}[k+1]$ with $B_{j}[l]$. Notice that in the above merging operation, we use the important feature that arcs in $B_{i}$ and $B_{j}$ are sorted according to their starting angles, and the merging operation can be done in sorted order.

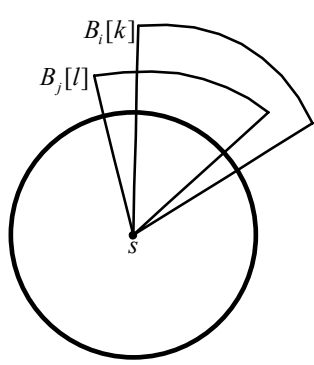

(a) Case 1.1

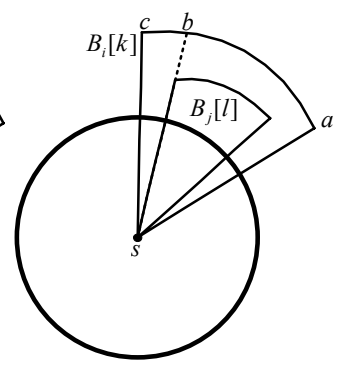

(b) Case 1.2

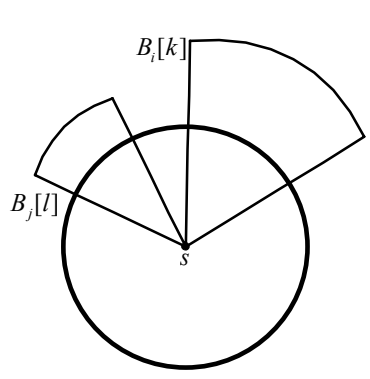

(c) Case 1.3

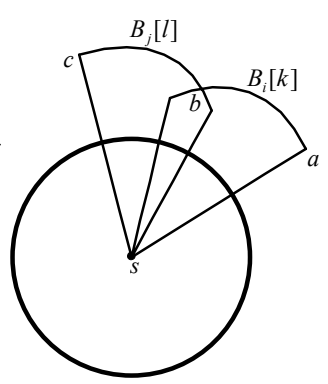

(d) Case 2

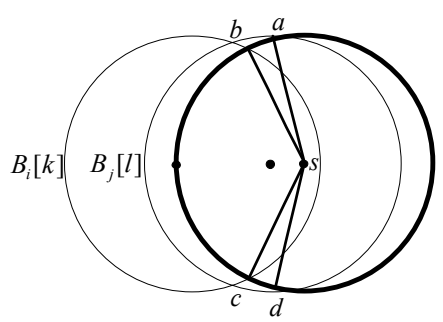

(e) Case 3

Fig. 5. Relationship between two arcs.

For the case where arcs $B_{i}[k]$ and $B_{j}[l]$ have only one intersecting point at $b$, as shown in Fig. 5(d), segment $a b$ of $B_{i}[k]$ contributes to $B$ and segment $b c$ of $B_{j}[l]$ may intersect 
with $B_{i}[k+1]$. Thus, we set $B[h]=a b$ and, then move to the next to compare $B_{i}[k+1]$ with the segment $b c$ of $B_{j}[l]$.

If arcs $B_{i}[k]$ and $B_{j}[l]$ have two intersecting points at $b$ and $c$, as shown in Fig. 5(e), both segments $a b$ of $B_{j}[l]$ and $b c$ of $B_{i}[k]$ contribute to $B$. We set $B[h]=a b$ and $B[h+1]=b c$. Then, we move to compare $B_{i}[k+1]$ with segment $e d$ of $B_{j}[l]$.

Following the above discussion of merging the two arcs in $B_{i}$ and $B_{j}$, and moving the pointer to the next arc for merging, this operation can be repeated until all arcs in $B_{i}$ are merged with $B_{j}$ into the new boundary $B$. The following is the detailed boundary merge algorithm.

\section{BoundaryMerge Algorithm \\ Input: $B_{i}$ and $B_{j}$. \\ Output: $B$. \\ Begin}

$k=1 ; / /$ pointer to the current $\operatorname{arc}$ in $B_{i}$.

$l=1 ; / /$ pointer to the current $\operatorname{arc}$ in $B_{j}$.

$h=1$; // pointer to the current $\operatorname{arc}$ in $B$.

while (there is unmerged arc in both $B_{i}$ and $B_{j}$ ) do

Merge $B_{i}[k]$ and $B_{j}[l]$ to $B$ according to cases $1-3$; Return $B$.

Adjust $k, l$, and $h$ accordingly;

\section{End}

Theorem 2. Time complexity of BoundaryMerge algorithm is $O\left(n_{1}+n_{2}\right)$, where $n_{1}$ and $n_{2}$ are the numbers of arcs in $B_{i}$ and $B_{j}$, respectively.

Proof. Notice that in BoundaryMerge algorithm, we always move to the next arc of $B_{i}$ or $B_{j}$ after comparison and no backtracking is needed. So the total running time is $O\left(n_{1}+n_{2}\right)$, where $n_{1}$ and $n_{2}$ are the number of arcs in $B_{i}$ and $B_{j}$, respectively. Theorem proved.

Now, we consider the forwarding node selection algorithm. Initially, for each node $i, 1 \leq i \leq|N(s)|$, its arc outside of the area of $d(s)$ is represented by a boundary array $B_{i}[]$. Then, the arcs are merged in pair-wise by using the BoundaryMerge algorithm, until there becomes a single boundary of the coverage area of $N(s) . F(s)$ consists of the nodes that contribute to this boundary.

\section{FwdNodes Algorithm \\ Input: $s$ and $N(s)$. \\ Output: $F(s)$. \\ Begin}

$$
\begin{aligned}
& j=n ; / / n=|N(s)| . \\
& \text { while } j>1 \text { do } \\
& \quad \text { for }(i=1 ; i<j ; i=i+2) \\
& \quad B_{(i+1) / 2}[]=\operatorname{BoundaryMerge}\left(B_{i}[], B_{i+1}[]\right) ; \\
& \quad j=j / 2 ;
\end{aligned}
$$

\section{End}

Output $F(s)=\left\{B[i] . u_{i} \mid i=1,2, \ldots, k\right\} ; / / B$ : the final boundary.

Notice that if $n$ is odd in the above algorithm, we add a virtual arc whose starting angle and ending angle are both $0^{\circ}$. It does not affect correctness of the output.
Theorem 3. Time complexity of FwdNodes algorithm is $O(n \log n)$, where $n=|N(s)|$.

Proof. In FwdNodes algorithm, each time we partition the current $n$ boundaries into $n / 2$ group, and run BoundaryMerge algorithm to merge two boundaries in each group. According to Theorem 2, it takes $O(n)$ to complete boundary merge in all groups. Since each time the number of groups reduces half, it costs $O(\log n)$ to obtain the final one group, i.e., coverage's boundary. So the total time complexity is $O(n \log n)$.

\section{Theorem 3 is proved.}

FwdNodes algorithm requires that each node has 1-hop information. According to Theorem 1, it guarantees that all nodes can receive the flooding message. Based on these conditions, the following theorem states that our algorithm is optimal.

Theorem 4. FwdNodes algorithm achieves local optimality in terms of: 1) the number of forwarding nodes is the minimal, i.e., $F(s)=F_{\min }(s)$; 2) the time complexity is the lowest.

Proof. In FwdNodes algorithm, each node only has 1-hop information. To cover 2-hop neighbors that are beyond its view, each node should select some 1-hop neighbors to relay the message, such that these selected neighbors can sufficiently cover the neighbor's area of the node. Thus, all 2hop neighbors are guaranteed to be covered. In the algorithm, each node $s$ selects the minimal set of nodes $F(s)$ to forward the message by computing the neighbor's boundary of $s$. Notice that any node in $F(s)$ contributes to the final boundary. If a node in $F(s)$ does not relay the message, other nodes can not take over its duty. It means that all nodes in $F(s)$ should forward the message to guarantee 2-hop neighbors are covered. Thus, we have $F(s)=F_{\text {min }}(s)$.

According to Theorem 3, time complexity of computing $F(s)$ is $O(n \log n)$, where $n=|N(s)|$. Notice that computing $F(s)$ is equivalent to computing the neighbor's boundary of $s$. We will prove that sorting problem can be induced to boundary computing problem.

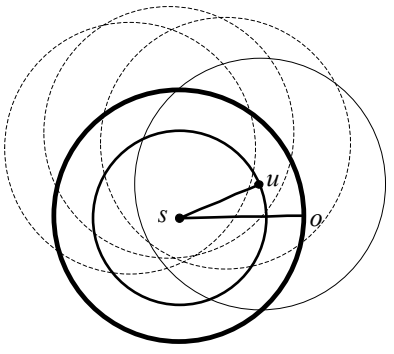

Fig. 6. Sorting problem is induced to boundary computing problem.

Given $n$ real numbers to be sorted, we first scale them to the numbers in $[0,2 \pi]$. For each scaled number $a \in[0,2 \pi]$, point $u$ is placed on the border of a small circle, such that $\angle u s o=a$ (See Fig. 6, centre of the small circle is $s$, line $o s$ is parallel to $X$-axis and angles are counted in anti-clock direction). Notice that these nodes are in $N(s)$ and the distance from $s$ are the same. So each disk of node contributes to the 
neighbor's boundary of $s$. After running FwdNodes algorithm, starting angles $B[i] . \theta_{i}^{s}, i=1,2, . . n$, of final boundary are in nondescending order. It is equivalent to sort the given $n$ nodes. That is, sorting problem can be induced to boundary computing problem. We know that the fastest sorting algorithm costs $O(n \log n)$ time. So FwdNodes algorithm is the fastest algorithm to compute the neighbor's boundary of $s$. Theorem 4 is proved.

After computing $F(s)$ by FwdNodes algorithm, $s$ attaches IDs of nodes in $F(s)$ to the flooding message and broadcasts it out. When receiving this message, a neighbor node of $s$ checks if its own ID is in the forwarding list attached to the message. If yes, it will call the FwdNodes algorithm and forward the message out, the same as $s$ does. In this way, the message is forwarded hop by hop until all the nodes in the network receive it.

\subsection{Forwarding Node Optimization}

The $F(s)$ computed above is only locally optimal based on the 1-hop information of $s$. When a node $u$ receives the flooding message from $s$ (we call $s$ the parent of $u$ ) and $u$ is a forwarding node nominated by $s$ (i.e., $u \in F(s)$ ), the computing of $F(u)$ can be further optimized based on the information of $F(s)$, which is attached to the flooding message from $s$. This is because some nodes in $F(u)$ may be already covered by node $s$ or node-set $F(s)$, and thus $F(u)$ could be further reduced by removing out those nodes.

Consider the example given in Fig. 7, where nodes $u$ and $v$ are neighbors of $s$ and $F(s)=\{u, v\}$. The coverage area $d(u)$ overlaps with $d(s)$ and $d(v)$ (notice node $v$ is also in $F(s)$ ). The nodes in the overlapped area of $d(u)$ with $d(s)$ were already considered by $s$ when computing $F(s)$. Thus, these nodes can be removed from $F(u)$. For the overlapped area of $d(u)$ with other nodes in $F(s)$, for example node $v$ in Fig. 7, we use node ID as the priority for forwarding messages. That is, the node with the smaller ID has to forward the message if its coverage disk overlaps with another node. Therefore, the nodes of $F(u)$ that fall into the coverage area of the following node-set can be removed from $F(u)$ :

$$
\{s\} \cup\{v \mid v \in(F(s) \cap N(u)) \text { and } i d(v) \leq i d(u)\} .
$$

Notice that in node-set (1), we only consider set $F(s) \cap$ $N(u)$. That is, nodes in the node-set (1) are all neighbors of node $u$. Thus, $u$ knows the geographic locations of nodes in the above node-set. This location information is necessary when $u$ checks whether nodes in $F(u)$ fall into the coverage area of the node-set (1). We can see this optimization is still based on 1-hop information of a node.

Taking the example in Fig. 7 again, suppose $i d(v) \leq i d(u)$ and $F(u)=\{1,2,3,4,5\}$. Since nodes 1 and 2 are in $N(s)$, they are already covered by $s$ and can be removed from $F(u)$. Node 3 is covered by $v$, and $v$ is also a forwarding node and $i d(v) \leq i d(u)$. Thus, node 3 can also be removed from $F(u)$. Finally, $F(u)=\{4,5\}$. That is, node $u$ only needs to nominate the nodes of $F(u)$ in clear area.

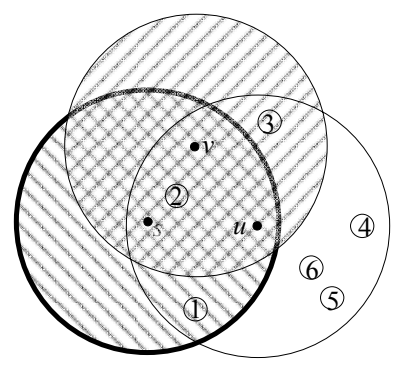

Fig. 7. An example of optimizing $F(u)$.

The significance of this optimization is that it prevents the flooding message from going backwards. The message is always propagated forward towards the uncovered area, which reduces the redundant transmissions greatly.

The following is the optimized forwarding node selection algorithm. It is executed whenever a node receives a flooding message.

\section{OptFwdNodes Algorithm}

Input: message $m$ from $s$.

Begin

if $m$ was received before, then discard $m$;

else

Deliver $m$ to upper layer;

if this node, say $u$, is in forward-list in $m$ Compute $F(u)$;

Remove from $F(u)$ the nodes that are covered by node-set (1);

Attach $F(u)$ to $m$ and transmit $m$ out.

\section{End}

According to Theorem 4, we know that FwdNodes algorithm can guarantee that all nodes receive flooding message. After optimization in the OptFwdNodes algorithm, the $100 \%$ deliverability feature is still preserved. The following theorem states this feature.

Theorem 5. The OptFwdNodes algorithm guarantees that all nodes can receive flooding message.

Proof. According to Theorem 4, if all forwarding nodes run FwdNodes algorithm, 100\% deliverability is guaranteed. So, to prove theorem 5, we need to prove that removing nodes from $F(u)$ in OptFwdNodes algorithm does not affect $100 \%$ deliverability of our scheme.

We suppose $\{u, v\} \subseteq F(s)$ and $i d(v) \leq i d(u)$. If some nodes in $F(u)$ are neighbors of $s$ and $v$, the coverage disks of these nodes are sure to be within the coverage area of $F(s)$ and $F(v)$. So there is no need to let these nodes forward the message. Thus, removing these nodes from $F(u)$ does not affect $100 \%$ deliverability of our scheme. Theorem is proved.

Time complexity of OptFwdNodes algorithm is given bellow.

Theorem 6. Time complexity of OptFwdNodes algorithm is $O(n \log n)$, where $n=|N(s)|$. 
Proof. It is not difficult to see the time complexity of OptFwdNodes algorithm is the same as that of FwdNodes algorithm. Theorem 6 is proved.

Source node first floods a message by running FwdNodes algorithm. Each forwarding node forwards the message by running OptFwdNodes algorithm. Finally, all nodes in the network can receive the message and the redundant transmission can be significantly reduced.

\section{MoBility HandLING}

In MANETs, nodes may be mobile, which causes dynamic changes of the network topology. For the flooding scheme, each node, say $s$, maintains its neighbor information and computes $F(s)$. To cope with the dynamic topology changes, there are two strategies to maintain the flooding scheme: a) No update. Each node re-computes its forwarding node set for each flooding request; or b) Incremental update. Each node incrementally updates its forwarding node set upon each topology change. For strategy (a), we do not need to do anything. In this section, we propose an efficient algorithm that can incrementally update the forwarding node set as the topology changes. By using this method, nodes do not need to re-compute the forwarding node set when it needs to flood a message. The forwarding node set is maintained at each node and is always ready for use.

For each node $u$, there are three cases that require updating $F(u): 1)$ a neighbor of $u$ moves but still in $N(u)$; 2) a neighbor of $u$ moves out of $N(u) ; 3)$ a node moves in and becomes the new neighbor of $u$. We assume that only one update is handled at a time. We concentrate on updating $F(u)$ for these three cases, and discuss them case by case.

Case 1. We consider the first case that the location of a neighbor $v$ changes but $v$ is still in $N(u)$. There are two subcases.

Case 1.1. If $v \notin F(u)$, we need to check whether the coverage disk of $v$ exceeds the neighbor's boundary of $u$. If it happens, disk of $v$ will contribute to the final boundary $B$ and $F(u)$ will be updated. We first compute how many arcs in $B$ are affected by the movement of $v$. It is can be done by locating the starting angle and ending angle of current location of disk $v$ in $B$ by binary search. Suppose that $k$ arcs in $B$ are affected by the arc of disk $v$. It means that the sectors of these arcs overlap with the sector of disk $v$. Notice that these $k$ arcs form a continuous segment of $B$ in non-decreasing order according to their starting angles. Then, we run BoundaryMerge algorithm to merge this segment and the arc of disk $v$ to update the new boundary $B$ and $F(u)$.

Case 1.2. If $v \in F(u)$, the final boundary $B$ not only may be affected by the current location of $v$, but also may be affected by the former location of $v$. Notice that $v \in F(u)$ and location of $v$ changes. Some nodes in $N(u)-F(u)$ may contribute to $B$ because $v$ leaves its former place. On the other hand, some nodes in $F(u)$ may become invalid because $v$ moves to the current place. So it has two steps update. We first compute how many arcs in $N(u)$ may contribute to the new boundary because of leaving of $v$. Since there is no order in $N(u)$, we find $k$ arcs that may contribute to $B$ one by one. We compute the new boundary of these $k$ arcs. Second, similar to case 1.1, we still need to compute how many arcs in $B$ are affected by the new location of $v$. Suppose $l$ continuous arcs in $B$ are affected. We update $B$ and $F(u)$ again by merging these $l$ continuous arcs and the arc of disk $v$ in current place.

Case 2. We consider the case that $v$ is a neighbor of $u$, and $v$ moves out of $N(u)$. If $v \notin F(u)$, there is no need to update. If $v \in F(u)$, some nodes in $N(u)-F(u)$ may contribute to $B$ due to the leaving of $v$. This is similar to the first step of case 1.2. We can update $F(u)$ for this case.

Case 3. We consider the case that a node $v$ moves into coverage disk of $u$, and becomes the new neighbor of $u$. Similar to case 1.1. We can update $F(u)$ for this case.

Detailed algorithm is given bellow.

\section{TopologyUpdate Algorithm}

Input: $v$ that changes its location to $u$.

Output: updated $F(u)$.

Begin

if $v \notin F(u)$ and $v$ is now in $N(u) / /$ case 1.1 or case 3 .

Find $\operatorname{arcs}$ in $B$ that are affected by disk $v$;

//suppose $k$ arcs $B[i], B[i+1], \ldots, B[i+k-1]$ are affected.

BoundaryMerge $(\{B[i], B[i+1], \ldots, B[i+k-1]\}, d(v))$;

if $v \in F(u) / /$ case 1.2 or case 2 .

Find arcs in $N(u)$ that are affected by $v$ 's leaving;

// suppose $k$ arcs are affected.

Compute the boundary of the affected $k$ arcs;

Find $\operatorname{arcs}$ in $B$ that are affected by $v$ 's current place;

// suppose $l$ arcs $B[i], B[i+1], \ldots, B[i+l-1]$ are affected.

BoundaryMerge $(\{B[i], B[i+1], \ldots, B[i+l-1]\}, d(v))$; End

Update $F(u)$ based on the new boundary $B$

Theorem 7. Time complexity of update for case 1.1 , case 3 and case 1.2 , case 2 are $O(k+\log n)$ and $O(n+k \log k)$, respectively, where $n=|N(s)|$ and $k$ is the number of nodes that are affected by topology change.

Proof. For case 1.1 and case 3 , it costs $O(\log n)$ to locate the arc of disk $v$ in $B$ by binary search. It further costs $O(k)$ to merge $\{B[i], \quad B[i+1], \ldots, B[i+k-1]\}$ and disk $v$ by BoundaryMerge algorithm. So the total time cost of update for case 1.1 and case 3 is $O(k+\log n)$.

For case 1.2 and case 2 , it costs $O(n)$ to find $k$ disks of nodes in $N(u)$ that are affected by movement of $v$. Similar to boundary computing in FwdNodes algorithm, computing new boundary of these $k$ disks costs $O(k \log k)$. It further costs $O(l+\log n)$ to compute the new boundary of $l$ disks in the second step. So the total time cost of update for case 1.2 and case 2 is $O(n+k \log k)$. Theorem 7 is proved.

From Theorem 7, we can see that update for case 1.1 and case 3 is very efficient comparing to re-computing $F(u)$. Update for case 1.2 and case 2 is also efficient when $k$ is not 
large. If $k=\Theta(n)$, time complexity of TopologyUpdate algorithm is the same as that of FwdNodes algorithm.

\section{SimULATION}

To analyze the performance of our flooding scheme, we compare it with three deliverability-guaranteed schemes: Pure flooding, Edge Forwarding (it requires 1-hop information [9]), and CDS-based flooding [11] (it requires 2-hop information). In CDS-based scheme, a node marks itself belonging to the CDS if there exist two unconnected neighbors. A marked node can quit the CDS later if its neighbors are covered by two CDS neighbors and they have greater IDs. It was proved that the marked nodes form a CDS [11]. Notice that all forwarding nodes in a flooding operation form a CDS in the network. It means that the number of forwarding nodes is no less than the number of MCDS (Minimum CDS) in the network. So the number of MCDS is the lower bound of the number of forwarding nodes. Although computing MCDS is NP-hard, there exists a ratio-8 approximation algorithm [29]. This lower bound is computed and is used as a benchmark for comparison with the simulated flooding schemes.

We study the performance of flooding schemes against two parameters: number of nodes, transmission range. We run simulations under the $n s-2$ test bed with the CMU wireless extension. The simulator parameters are listed in Tab. 1. The popular two-ray ground reflection model is adopted as the radio propagation model. The MAC layer scheme follows the IEEE 802.11 MAC specification. We use the broadcast mode with no RTS/CTS/ACK mechanisms for all message transmissions. Each data packet with attached information has a constant length of 256 bytes. The bandwidth of a wireless channel is set to $2 \mathrm{M} \mathrm{b} / \mathrm{s}$ as default. Some of the schemes require nodes to send HELLO message to their 1-hop neighbors periodically. This cost of HELLO message is ignored in our performance study.

TAB. 1. SIMULATION PARAMETERS.

\begin{tabular}{|c|c|}
\hline Parameter & Value \\
\hline Simulator & $n s-2$ (version 2.28$)$ \\
\hline MAC Layer & IEEE 802.11 \\
\hline Data Packet Size & 256 bytes \\
\hline Bandwidth & $2 \mathrm{Mb} / \mathrm{s}$ \\
\hline Transmission Range & $100 \sim 300$ meter \\
\hline Number of Node & $200 \sim 1000$ \\
\hline Size of Square Area & $200,000 \sim 1,000,000$ meter $^{2}$ \\
\hline Number of Trails & 100 \\
\hline
\end{tabular}

The main objective of those efficient flooding schemes is to reduce the number of forwarding nodes as much as possible, such that the redundant transmission is minimized. So we use the metric ratio of forwarding nodes to evaluate the efficiency of flooding schemes. The ratio of forwarding nodes is defined to be the ratio of total number of nodes involved in the packet forwarding in a flooding operation over the total number of nodes in the network, such as: ratio of forwarding nodes $=\frac{\text { the number of forwarding nodes }}{\text { the number of total nodes }}$.

Reducing the forwarding nodes in flooding would effectively reduce the signal collision in the network. The MAC layer of IEEE 802.11 in $n s-2$ can check the occurrence of collisions. If the number of collisions is high, it would result in more packet loss or more retransmissions. We also use the metric, number of collisions, to evaluate the efficiency of flooding schemes. The number of collisions is defined to be the sum of collisions that each node experiences before it receives the flooding message correctly.

Signal collisions will eventually affect the deliverability of flooding messages. Some nodes in the network miss flooding messages due to the large number of collisions. The metric, deliverability ratio, is used to further study the efficiency of algorithms. The deliverability ratio is defined by the number of nodes that successfully receive the flooding messages over the total number of nodes in the network.

In each simulation run, we generate a certain number of nodes and randomly place them on a square area. There is a link between two nodes if and only if their Euclidean distance is not greater than transmission range $R$. The source which initiates a flooding message is randomly picked from nodes in the network. Only one flooding occurs at any one time (except for the experiments of deliverability ratio). Three flooding schemes and the theoretical lower bound that are mentioned above are simulated and compared with our scheme under the same environment. We study how the ratio of forwarding nodes, the number of collisions and the deliverability ratio are affected by two parameters: the number of nodes, transmission range, respectively. The results presented in the following figures are the means of 100 separate runs. Any case where the network is not connected is discarded.

\subsection{Performance versus Number of Nodes}

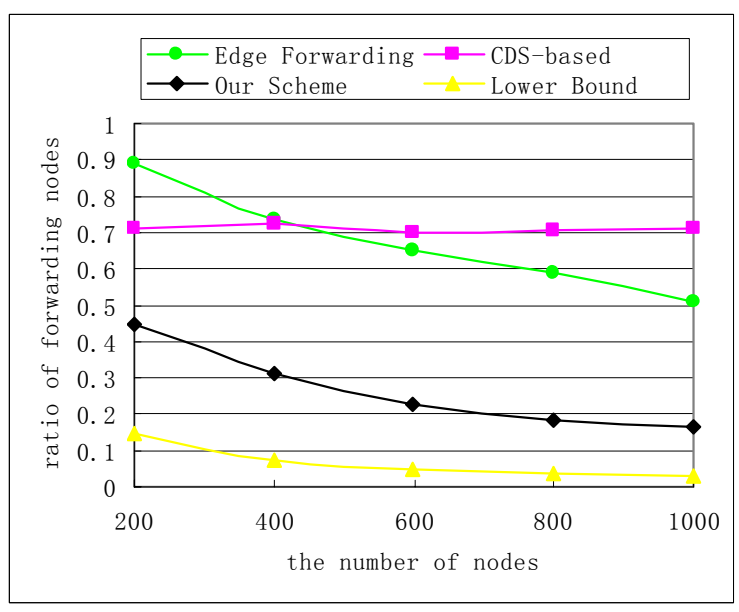

Fig. 8. Ratio of forwarding nodes VS. the number of nodes. 


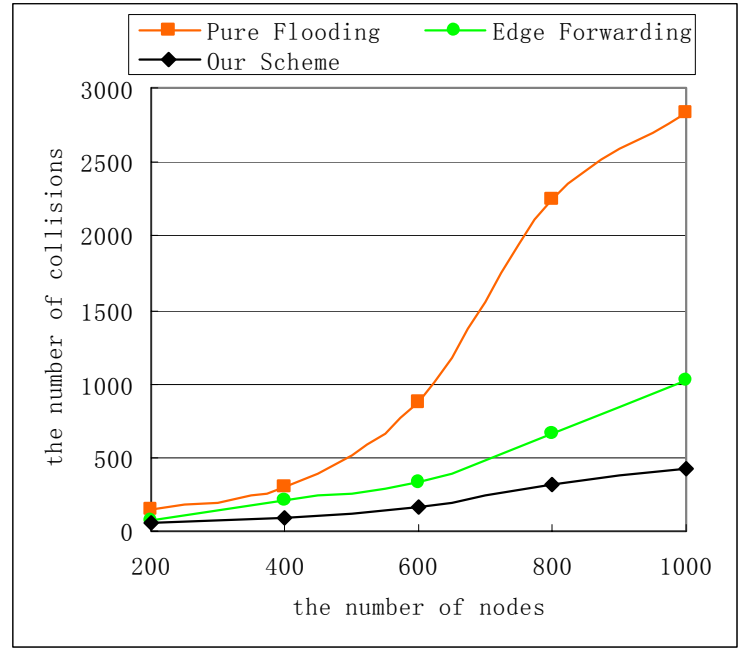

Fig. 9. The number of collisions VS. the number of nodes.

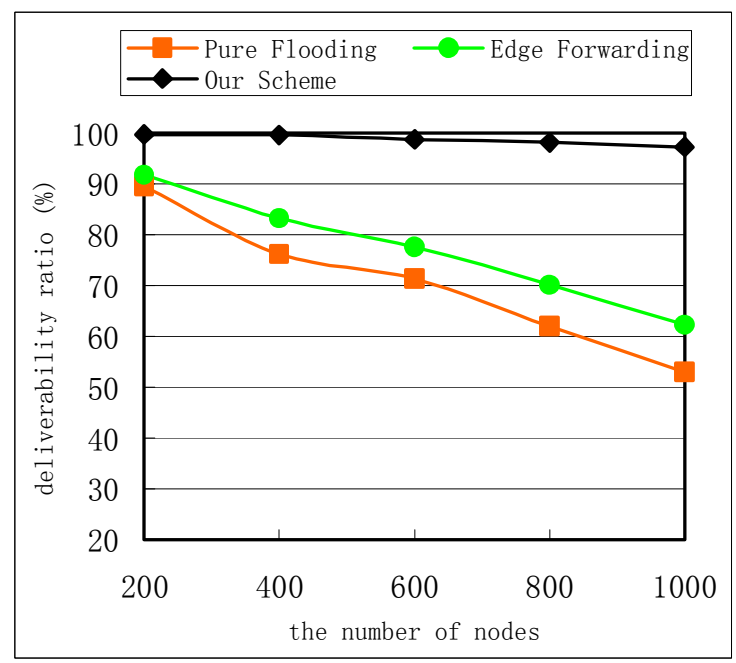

Fig. 10. Deliverability ratio VS. the number of nodes.

In this simulation, a certain number of nodes, from 200 to 1000 , are randomly placed on a $1000 \times 1000 \mathrm{~m}^{2}$ area. The transmission range is fixed at $250 \mathrm{~m}$. In the experiment of deliverability ratio in Fig. 10, the network load is set to $10 \mathrm{Pkt} / \mathrm{s}$. It means that the network generates 10 flooding messages per second on average. Deliverability ratio is calculated for 100 seconds. Since every node is a forwarding node in the pure flooding scheme, its curve was dropped out in Fig. 8. The simulation results are plotted in Fig. 8, Fig. 9 and Fig. 10. We have following observations.

1) Performance of our flooding scheme is significantly better than performance of Edge Forwarding and CDS-based schemes showed in Fig, 8. It is because that each forwarding node $u$ selects the minimal $F(u)$ to cover all 2hop neighbors in our scheme. It guarantees that the number of forwarding nodes is minimized at each step while Edge Forwarding and CDS-based schemes do not.

2) The curve of our scheme becomes closer to the curve of lower bound when the number of nodes increases. See Fig.
8 , when the number of nodes reaches 1000 , only $16.5 \%$ of nodes participate in forwarding in our scheme while ratios of Edge Forwarding and CDS-based schemes are 50.7\% and $71 \%$, respectively. This is because, as the increase of network density (resulting from the increase of nodes), $N(u)$ becomes larger, but $F(u)$ is saturated. That is, the number of nodes required to cover the same area (i.e., the neighbor's area) will not increase that much, because each node has a fixed coverage disk. Therefore, the ratio $F(u) / N(u)$ decreases as the increase of nodes in the network. We can conclude that our flooding scheme is more suitable for networks with high density.

3) Both the curves of our scheme and of Edge Forwarding fall down when the number of nodes increases in Fig. 8. But, number of nodes has little effect on the result of CDS-based scheme. Notice that when network density increases, there is more chance for $u$ 's neighbors being connected. At the same time, high density also causes increase of $N(u)$. It means there is a high chance that there exist two unconnected neighbors. So these two conflicting factors make the result of CDS-based scheme not sensitive to the change of number of nodes.

4) Our scheme and Edge Forwarding both have much lower collisions comparing with pure flooding. The reason is that every node forwards flooding messages in pure flooding while our scheme and Edge Forwarding only select a subset of neighbors to forward the messages. This smaller set of forwarding nodes will result in less collisions in the network. Performance of our scheme is better than that of Edge Forwarding. For example in Fig. 9 , when the number of nodes reaches 600 , the number of collisions of our scheme is only 211 while that of Edge Forwarding schemes are 364. After that, the collisions of Edge Forwarding method is over $100 \%$ higher than our scheme.

5) Deliverability ratio of our scheme is significantly higher than the ratios of Edge Forwarding and Pure Flooding. See Fig. 10, our scheme guarantees $100 \%$ deliverability when the number of nodes varies from 200 to 400 while deliverability ratios of Edge Forwarding and Pure Flooding are only $75 \%-90 \%$ around. Although collisions occur in our scheme even the number of nodes is small, a node that misses flooding messages from a forwarding node still has chance to receive messages from another forwarding node. So the value of our scheme can almost reach $100 \%$ if the number of nodes is between 200 to 600 (the number of collisions is low). The performance of Pure Flooding is the worst among three schemes. It is caused by the broadcast storm problem since every node re-transmits the flooding message in the network. 


\subsection{Performance versus Transmission Range}

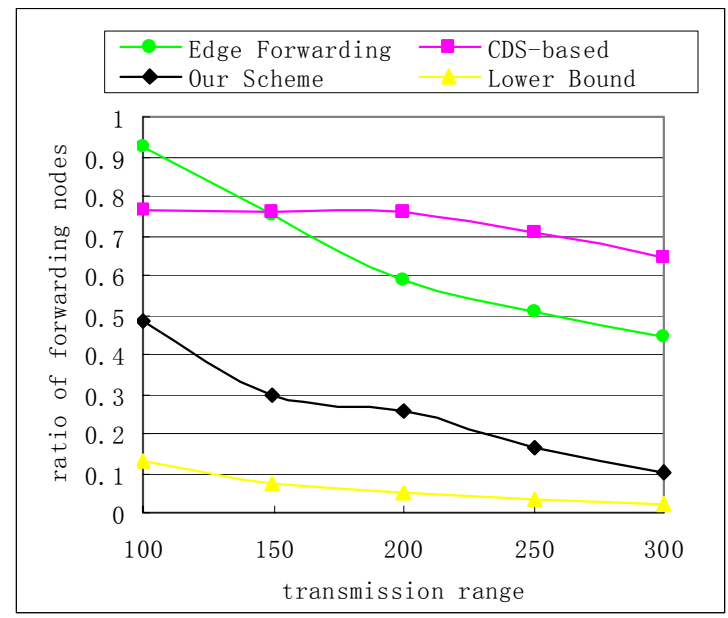

Fig. 11. Ratio of forwarding nodes VS. transmission range

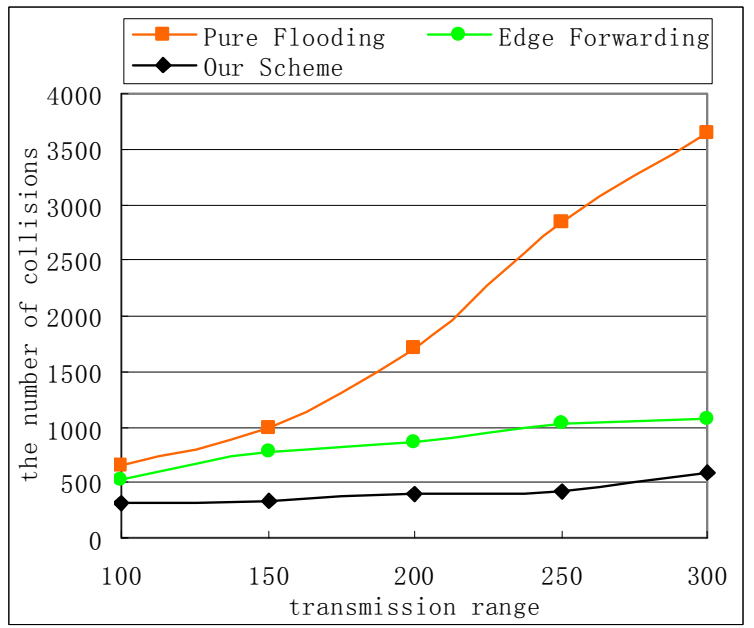

Fig. 12. The number of collisions VS. transmission range.

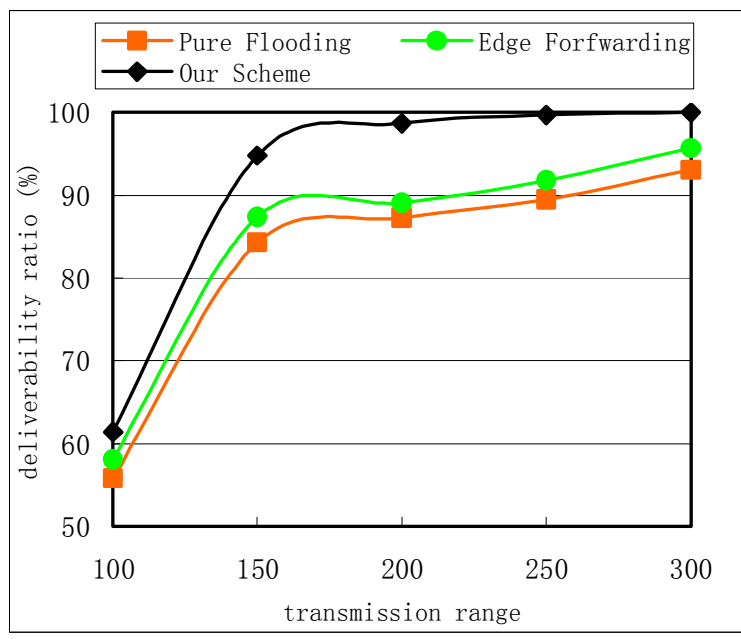

Fig. 13. Deliverability ratio VS. transmission range.

In this simulation, 1000 nodes are randomly placed on a $1000 \times 1000 \mathrm{~m}^{2}$ area. The network load is set to $10 \mathrm{Pkt} / \mathrm{s}$ and each simulation is run for 100 seconds in Fig. 13. We study the performance against the transmission range of each node. The simulation results are plotted in Fig. 11, Fig. 12 and Fig. 13. We have following observations.

1) Performance of our scheme is significantly better than performance of Edge Forwarding and CDS-based schemes shown in Fig. 11. The reason is similar to the results in Fig. 8. As the increase of transmission range, each node has more neighbors. It has the same effect on the increase of network density as the increase of nodes in a fixed square area.

2) The curve of our scheme becomes closer to the curve of lower bound when transmission range increases in Fig. 11. This trend is more significant than that in Fig. 8. See Fig. 11 , when the transmission range reaches $300 \mathrm{~m}$, only $10.4 \%$ of nodes participate in forwarding in our flooding scheme while values of Edge Forwarding and CDS-based schemes are $44.6 \%$ and $64.6 \%$, respectively. This is because that increase of transmission range not only results in higher density of network, but also makes flooding faster in the network. It means that flooding operation can be done in less steps due to the large transmission range of nodes. Notice that our scheme achieves that the number of forwarding nodes is minimal at each step. So less steps to complete flooding makes our results closer to the lower bound when transmission range increases.

3) Both the curves of our scheme and Edge Forwarding fall down when transmission range increases in Fig. 11. The curve of CDS-based scheme does not change much when $R$ increases from $100 \mathrm{~m}$ to $200 \mathrm{~m}$. The reason has been discussed before. But further increase of $R$ makes the curve fall down. It is because that when $R$ reaches a certain value, such as $200 \mathrm{~m}$ in Fig. 11, further increase of $R$ will slightly increase $N(u)$ due to the fixed number of nodes. But increase of $R$ makes nodes have more chance to be connected. So curve of CDS-based scheme falls down when $R$ is more than $200 \mathrm{~m}$.

4) Curves in Fig. 12 show the similar trend as those in Fig. 9. When the transmission range increases (i.e., a node has more neighbors), there are more chances for nodes to experience collisions. Since our scheme minimizes the number of forwarding nodes in each step, its performance is much better than that of pure flooding and Edge Forwarding schemes.

5) Deliverability ratios of three schemes all increase when transmission range increases in Fig. 13. It is because that increase of transmission range not only causes more collisions, but also provides more chances for nodes to receive flooding messages from different forwarding nodes. See Fig. 13, the ratio of our scheme becomes very close to $100 \%$ after transmission range is larger than 200 . Our scheme performs best among three schemes. 


\section{CONCLUSIONS}

The paper addressed the efficient flooding problem in MANETs. We have presented an efficient flooding scheme that uses only 1-hop neighbor information. We have proved that our proposed scheme achieves the local optimality in terms of: 1) the number of forwarding nodes is the minimal; 2) the time complexity $O(n \log n)$ is the lowest. Extensive simulations have been conducted to compare our scheme with pure flooding, Edge Forwarding and CDS-based schemes. Simulation results have shown that our proposed scheme uses less forwarding nodes, incurs less collision, obtains high deliverability ratio, compared with the existing schemes.

\section{REFERENCES}

[1] D. Johnson and D. A. Maltz, "Dynamic Source Routing in Ad Hoc Wireless Networks," in Mobile Computing, T. Imielinski and H. F. Korth, Eds., pp. 153-181. Kluwer Academic Publishers, Dordrecht, The Netherlands, 1996.

[2] C. E. Perkins, "Ad Hoc On-Demand Distance Vector (AODV) Routing," INTERNET DRAFT - Mobile Ad hoc NETworking (MONET) Working group of the Internet Engineering Task Force (IETF), November 1997.

[3] Z. J. Haas and M. R. Pearlman, "The Zone Routing Protocol (ZRP) for Ad Hoc Networks," INTERNET DRAFT - Mobile Ad hoc NETworking (MONET) Working group of the Internet Engineering Task Force (IETF), November 1997.

[4] Y. Ko and N. Yaidya, "Location-Aided Routing (LAR) in Mobile Ad Hoc Networks," in Proc. of MOBICOM'98, 1998, pp. 66-75.

[5] C. Ho, K. Obraczka, G. Tsudik, and K. Viswanath, "Flooding for Reliable Multicast in Multi-hop Ad Hoc Networks," in Proc. of the Int'l Workshop on Discrete Algorithms and Methods for Mobile Computing and Communication, 1999, pp. 64-71.

[6] J. Jetcheva, Y. Hu, D. Maltz, and D. Johnson, "A Simple Protocol for Multicast and Broadcast in Mobile Ad Hoc Networks," Internet Draft: draft-ietf-manet-simple-mbcast-01.txt, July 2001.

[7] S. Ni, Y. Tseng, Y. Chen, and J. Sheu, "The broadcast storm problem in a mobile ad hoc network," Proc. of ACM/IEEE MOBICOM'99, pp. 151162, Aug. 1999.

[8] P. Sinha, R. Sivakumar and V. Bharghavan, "Enhancing ad hoc routing with dynamic virtual infrastructures", IEEE INFOCOM 2001, pp. 17631772.

[9] Ying Cai, Kien A. Hua, and Aaron Phillips, "Leveraging 1-hop Neighborhood Knowledge for Efficient Flooding in Wireless Ad Hoc Networks," $24^{\text {th }}$ IEEE International Performance Computing and Communications Conference (IPCCC), April 7-9, 2005, Phoenix, Arizona.

[10] Chun-Chuan Yang and Chao-Yu Chen, "A Reachability-Guaranteed Approach for Reducing the Broadcast Storms in MANETs," Proceedings of IEEE Semiannual Vehicular Technology Conference (VTC-2002 Fall), Sept. 2002.

[11] J. Wu and H. Li, "On Calculating Connected Dominating Set for Efficient Routing in Ad Hoc Wireless Networks," in Proc. of the 3rd Int'l Workshop on Discrete Algorithms and Methods for Mobile Computing and Communications (DiaLM), 1999, pp. 7-14.

[12] J.E. Wieselthier, G. D. Nguyen, and A. Ephremides, "On the Construction of Energy-Efficient Broadcast and Multicast Trees in Wireless Networks", IEEE Infocom'2000.
[13] Deying Li, Xioahua Jia and Hai Liu, "Energy efficient broadcast routing in ad hoc wireless networks", IEEE Trans on Mobile Computing, Vol. 3, No. 2, Apr - Jun, 2004, pp.144-151.

[14] Y. Tseng, S. Ni, and E. Y. Shih, "Adaptive Approaches to Relieving Broadcast Storms in a Wireless Multihop Mobile Ad Hoc Networks," In Proc. of ICDCS'01, pp 481-488, 2001.

[15] Y. Sasson, D. Cavin, and A. Schiper, "Probabilistic broadcast for flooding in wireless mobile ad hoc networks," In Swiss Federal Institute of Technology, Technical Report IC/2002/54, 2002.

[16] M. T. Sun, W. C. Feng, and T. H. Lai, "Location Aided broadcast in wireless ad hoc networks," in Proc. of GLOBECOM'01, 2001.

[17] H. Lim and C. Kim, "Multicast Tree Construction and Flooding in Wireless Ad Hoc Networks," In Proc. of the ACM Int'l Workshop on Modeling, Analysis and Simulation of Wireless and Mobile System (MSWIM), pp 61-68, Aug. 2000.

[18] B. Williams and T. Camp, "Comparison of Broadcasting Techniques for Mobile Ad Hoc Networks," In Proc. of MOBIHOC'02, pp 914-205, 2002.

[19] A. Laouiti, A. Qayyum, and L. Viennot, "Multipoint relaying: An efficient technique for flooding in mobile wireless networks," In $35^{\text {th }}$ Annual Hawaii International Conference on System Sciences (HICSS'2001). IEEE Computer Society, 2001.

[20] A. Qayyum, L. Viennot, and Anis Laouiti, "Multipoint Relaying for Flooding Broadcast Messages in Mobile Wireless Networks," In Proceeding of the $35^{\text {th }}$ Hawaii International Conference on System Sciences, 2002.

[21] Wei Lou and Jie Wu, "Double-Covered Broadcast (DCB): A Simple Reliable Broadcast Algorithm in MANETs," in Proc. of INFOCOM 2004, 2004.

[22] B. Chen, K. Jamieson, H. Balakrishnan, and R. Morris, "Span: An Energy-Efficient Coordination Algorithm for Topology Maintenance in Ad Hoc Wireless Networks," in Proc. of MOBICOM'01, July 2001, pp. 85-96.

[23] W. Peng and X. Lu, "On the Reduction of Broadcast Redundancy in Mobile Ad Hoc Networks," in Proc. of MOBIHOC'00, 2000.

[24] I. Stojmenovic, M. Seddigh, and J. Zunic, "Dominating Sets and Neighbor Elimination Based Broadcasting Algorithms in Wireless Networks," IEEE Transactions on Parallel and Distributed Systems, vol. 13, no. 1, pp. 14-25, January 2002.

[25] J. Sucec and I. Marsic, "An Efficient Distributed Network-Wide Broadcast Algorithm for Mobile Ad Hoc Networks," in Rutgers University, CAIP Technical Report 248, September 2000.

[26] J. Wu and F. Dai, "Broadcasting in Ad Hoc Networks Based on SelfPruning," in Proc. of INFOCOM'03, March 2003.

[27] Fei Dai and Jie Wu, "An Extended Localized Algorithm for Connected Dominating Set Formation in Ad Hoc Wireless Networks," IEEE Trans. Parallel Distrib. Syst. 15(10): 908-920, 2004.

[28] M. V. Marathe, H. Breu, H. B. Hunt III, S. S. Ravi, and D. J. Rosenkrantz, "Simple heuristics for unit disk graphs," Networks, vol. 25 , pp 59-68, 1995.

[29] P.-J. Wan, K. Alzoubi, and O. Frieder, "Distributed Construction of Connected Dominating Set in Wireless Ad Hoc Networks," Proc. IEEE INFOCOM, vol. 3, pp 1597-1604, June 2002.

[30] Dariusz R. Kowalski and Andrzej Pelc, "Deterministic Broadcasting Time in Radio Networks of Unknown Topoloby," Proc. of the $43^{\text {rd }}$ Annual IEEE Symposium on Foundations of Computer Science (FOCS'02), 2002.

[31] Rajiv Gandhi, Srinivasan Parthasarathy, and Arunesh Mishra, "Minimizing Broadcast Latency and Redundancy in Ad Hoc Networks," In Proc. of the Fourth ACM Int. Symposium on Mobile Ad Hoc Networking and Computing (MOBIHOC'03), pp 222-232, Jun. 2003. 